\title{
Polifarmacia y calidad de vida en adultos mayores del Centro Residencial Beneficencia de Huancayo
}

\author{
Polypharmacy and quality of life in older adults in the Residential Center - Beneficencia de Huancayo
}

Mónica Alejandra Calle Vilca ${ }^{1, a}$, Diana Esmeralda Andamayo Flores ${ }^{1, b}$, Lizzy Jeanette Mendoza Gutierrez 1,c, Vilma Amparo Junchaya Yllescas ${ }^{1, d}$

\section{RESUMEN}

Objetivo: Determinar la relación de polifarmacia con la calidad de vida en adultos mayores del centro de atención residencial San Vicente de Paúl de la sociedad de beneficencia de Huancayo. Material y Métodos: El tipo de investigación es básica, descriptivo, transversal de diseño no experimental basado en la recopilación de información de las fichas clínicas y encuestas, la población fue de 40 adultos mayores. Resultados: Se encontró que existe relación entre la polifarmacia y la calidad de vida, la frecuencia de polifarmacia es del 92,5\%, el nivel de adherencia es de $100 \%$, la valoración global de la calidad de vida tiene un valor 54,83 . Conclusiones: La polifarmacia afecta la calidad de vida de los adultos mayores, se tiene una frecuencia elevada de polifarmacia y calidad de vida en un estado regular.

PALABRAS CLAVE: polifarmacia, calidad de vida, adulto mayor, adherencia

\section{SUMMARY}

Objective: To determine the relationship between polypharmacy and quality of life in older adults of the San Vicente de Paul residential care center of the Huancayo charitable society. Material and Methods: The research is basic, descriptive, cross-sectional, non-experimental design based on the collection of information from clinical records and surveys, the population was 40 older adults. Results: Was found that there is a relationship between polypharmacy and quality of life, the frequency of polypharmacy is $92.5 \%$, the level of adherence is $100 \%$, the overall assessment of quality of life has a value 54.83. Conclusions: The polypharmacy affects the quality of life of older adults.

KEY WORDS: Polypharmacy, quality of life, older adults, adherence.

\footnotetext{
Universidad Privada de Huancayo Franklin Roosevelt. Huancayo. Perú.

Docente; Químico Farmacéutico; Magister; ORCID ID: 0000-0001-9317-519X

Docente; Químico Farmacéutico; Magister; Doctor; ORCID ID: 0000-0003-3357-3537

Docente; Químico Farmacéutico; 'Magister; ORCID ID: 0000-0001-9121-6808

Docente; Químico Farmacéutico; 'Magister; ORCID ID: 0000-0002-5035-2157
} 


\section{INTRODUCCIÓN}

El adulto mayor siendo una persona vulnerable frente a diversas enfermedades, requiere tratamiento para cada una de ellas, por lo que puede presentarse polifarmacia en el tratamiento. La polifarmacia es el uso concurrente de múltiples medicamentos, aunque no hay una definición estándar, la polifarmacia se define a menudo como polifarmacia menor como el uso de 2 a 4 medicamentos y más de 5 como polifarmacia mayor (1-3). Es inevitable que el adulto mayor frente al tratamiento de sus enfermedades presente otras molestias como consecuencia de la polifarmacia, es así que puede afectar la calidad de vida de estos. La calidad de vida según la Organización Mundial de la Salud (OMS) puede ser definida como "la percepción individual de la posición en la vida en el contexto de la cultura y sistema de valores en el cual se vive y su relación con las metas, expectativas, estándares e intereses" (4). No solo la polifarmacia es un problema que afecta al adulto mayor en cuanto a la calidad de vida, sino también vayamos a ver el tema de adherencia farmacológica.

En el año 2003 la OMS define el término adherencia como "el grado en el que la conducta de un paciente, en relación con la toma de medicación, el seguimiento de una dieta o la modificación de hábitos de vida, se corresponde con las recomendaciones acordadas con el profesional sanitario" (5). En este aspecto muchos de los pacientes tienen dificultades para cumplir con las prescripciones médicas debido a los diferentes hábitos de vida, afectando a su salud de diferentes maneras, alterando su calidad de vida.

La polifarmacia entendiéndose como el uso simultaneo o excesivo de varios medicamentos que se viene dando en adulos mayores, pacientes geriátricos, se presenta como un problema de salud pública que puede conllevar a interacciones medicamentosas y por ende a reacciones adversas, en donde el anciano con múltiples comorbilidades es el más expuesto y siendo así la presente investigación se justifica con la finalidad de evaluar la incidencia de polifarmacia y cómo influye en la calidad de vida del paciente para que de esta manera se pueda brindar recomendaciones al médico prescriptor, químico farmacéutico, enfermeros y otros profesionales involucrados en la salud a los pacientes geriátricos y a la comunidad en general. La relevancia del estudio se basa en el objetivo de determinar la relación de la polifarmacia y la calidad de vida en adultos mayores del Centro de atención residencial
San Vicente de Paúl de la Sociedad de Beneficencia de Huancayo

Por lo tanto, los resultados obtenidos contribuirán a la detección de los problemas de polifarmacia y establecer políticas de salud en la atención del adulto mayor y poder mejorar su calidad de vida.

\section{MATERIAL Y MÉTODOS}

La investigación es de tipo básica, nivel descriptiva diseño no experimental relacional transversal. La información fue recogida de los residentes del Centro de atención residencial San Vicente de Paúl de la Sociedad de Beneficencia de Huancayo, de sus hojas clínica y la aplicación de encuestas. La población estuvo constituida por 40 adultos mayores y luego de la aplicación de los criterios de inclusión (adultos mayores de más de 60 años, de ambos sexos y que se encuentre en sano juicio) y criterios de exclusión (adultos de menos de 60 años, que no se encuentre en sano juicio) se trabajó con 20 adultos mayores para la determinación de la valoración de calidad de vida de los mismos. La técnica utilizada fue análisis documental y la encuesta; el instrumento para recoger la información fueron una ficha clínica, el Cuestionario MBG (Martin-Bayarre-Grau) para la determinación de la adherencia farmacológica y el instrumento SF36 modificado, cuestionario para medir la calidad de vida de los adultos mayores.

Las variables de interés son polifarmacia y calidad de vida, considerando que polifarmacia es el uso concurrente de múltiples medicamentos, polifarmacia menor al uso de hasta tres medicamentos y polifarmacia mayor al consumo de más de tres medicamentos (1-3). La calidad de vida en el adulto mayor es determinada por la valoración que ellos realizan de su bienestar físico, psíquico y social, que les permita dentro de su vida cotidiana la normalidad (6).

Se valoraron las dimensiones establecidas como: nivel de polifarmacia (polifarmacia mayor y polifarmacia menor), adherencia farmacológica (adherencia total 31 a 48 puntos, adherencia parcial 18 a 33 puntos y no adherencia menos de 17 puntos), grupos farmacológico prescritos (según grupos terapéutico del PNUME) y se valoró la calidad de vida (en las dimensiones de funcionamiento físico, bienestar emocional y funcionamiento social. Tomando como referencia una escala de 0 a 100). 
Como parámetros estadísticos se utilizaron la prueba de independencia de Chi cuadrado para la determinación de la relación entre variable. Así como la escala de percentiles donde se ha considerado buena, regular y mala, tomando el percentil 50 , considerando las categorías buena (mayor a 75\%), regular (oscila entre 50 y $75 \%$ ) y mala (menor de $50 \%$ )

El estudio fue aprobado por el Comité Institucional de ética en investigación de la Universidad Privada de Huancayo Franklin Roosevelt bajo la opinión de los miembros de Cómité con Acta $\mathrm{N}^{\circ}$ 0534-FARM-CIEI.

\section{RESULTADOS}

En la tabla 1 se aprecia que el $92,5 \%$ de frecuencia de polifarmacia en los adultos mayores de la Sociedad de Beneficencia de Huancayo, de los cuales el mayor porcentaje es de polifarmacia menor con un $72,5 \%$ y polifarmacia mayor sólo el $20 \%$.

En la tabla 2 se observa que el nivel de adherencia farmacológica fue del $100 \%$, siendo el nivel de adherencia total $70 \%$ y nivel adherencia parcial $30 \%$.

En la tabla 3 describe los grupos farmacológicos más prescrito, las Vitaminas y minerales con un 31\%, seguido de los medicamentos psicoterapéuticos con un $17 \%$, los medicamentosa cardiovasculares con un $14 \%$, los medicamentos que actúan en las vías respiratorias con $7 \%$ y analgésicos $6 \%$.

En los resultados de la tabla 4 sobre calidad de vida de los adultos mayores se observa que en la dimensión de funcionamiento sociales se ha alcanzado un valor de 70,63 que indica un estado regular, en bienestar emocional 58,08 indica un estado regular, funcionamiento físico 47,85 indica un estado malo, con un valor global de la calidad de vida de 54.83, el estado de la calidad de vida es regular según la escala de percentiles

Se realizó una prueba de independencia de chi-cuadrado para examinar la relación entre la polifarmacia y la adherencia farmacológica. El estadístico de chi-cuadrado es 16,2384. El valor $\mathrm{p}$ es 0,000298 , el resultado es significativo en $\mathrm{p}<0,05$, lo que indica que no existe relación entre las variables (tabla 5).

Se realizó una prueba de independencia de chi-cuadrado para examinar la relación entre la polifarmacia y la calidad de vida (dimensiones: función física, bienestar emocional y función social). El estadístico de chi-cuadrado es 1,6718. El valor $\mathrm{p}$ es 0,795832 . El resultado no es significativo en $p<0,05$, lo que indica que existe relación entre las variables (tabla 6).

Se realizó una prueba de independencia de chicuadrado para examinar la relación entre la adherencia farmacológica y calidad de vida (dimensiones: función física, bienestar emocional y función social), el estadístico de chi-cuadrado es 0,6805 . El valor $p$ es 0,711578 . El resultado no es significativo en $p<0,05$, lo cual indica que existe relación entre las variables (tabla 7).

Tabla 1. Nivel de polifarmacia

\begin{tabular}{lcc}
\hline & Frecuencia & Porcentaje \\
\hline Sin polifarmacia & 3 & $7,5 \%$ \\
Polifarmacia menor & 29 & $72,5 \%$ \\
Polifarmacia mayor & 8 & $20 \%$ \\
Total & 40 & 100 \\
\hline
\end{tabular}

Tabla 2. Adherencia farmacológica

\begin{tabular}{lcc}
\hline & Nivel de adherencia & Porcentaje \\
\hline Total & 28 & $70 \%$ \\
Parcial & 12 & $30 \%$ \\
No adherencia & 0 & $0 \%$ \\
Total & 40 & $100 \%$ \\
\hline
\end{tabular}

Fuente: autoras (cuestionario MBG) 
Tabla 3. Grupos farmacológicos utilizados

\begin{tabular}{lcc}
\hline & Frecuencia & Porcentaje \\
\hline Medicamentos Cardiovasculares & 19 & $14 \%$ \\
Vitaminas Y Minerales & 43 & $31 \%$ \\
Medicamentos Psicoterapéuticos & 23 & $17 \%$ \\
Analgésicos & 8 & $6 \%$ \\
Medicamentos Gastrointestinales & 8 & $6 \%$ \\
Med. que actuan en las vias Respiratorias & 9 & $7 \%$ \\
Antibioticos & 5 & $4 \%$ \\
Antiparkinsonianos & 6 & $4 \%$ \\
Urológicos & 5 & $4 \%$ \\
Lubricante Oftálmico & 7 & $5 \%$ \\
Hormona Tiroidea & 3 & $2 \%$ \\
Hipoglucemiante & 1 & $1 \%$ \\
Antineoplásico & 1 & $1 \%$ \\
\hline
\end{tabular}

Tabla 4. Calidad de vida

\begin{tabular}{lc}
\hline Dimensiones de Calidad de Vida & Valor \\
\hline Funcionamiento Físico & 47,85 \\
Limitaciones debido a salud física & 50,00 \\
Limitaciones debido a problemas emocionales & 26,67 \\
Energía/Fatiga & 57,25 \\
Bienestar Emocional & 58,08 \\
Funcionamiento Social & 70,63 \\
Dolor & 58,25 \\
Salud General & 58,50 \\
Cambio De Salud & 66,25 \\
Promedio & 54,83 \\
\hline Fuente: autoras (cuestionario SF-36)
\end{tabular}

Tabla 5. Polifarmacia y adherencia farmacológica

\begin{tabular}{lcccccc}
\hline & Adherencia total & $\mathbf{\%}$ & Adherencia parcial & $\mathbf{\%}$ & Total & $\mathbf{\%}$ \\
\hline Sin Polifarmacia & 2 & 5 & 1 & 2,50 & 3 & 7,50 \\
Polifarmacia Menor & 25 & 62,50 & 4 & 10 & 29 & 72,50 \\
Polifarmacia Mayor & 1 & $2 ., 50$ & 7 & 17,50 & 8 & 20 \\
Totales de columna & 28 & 70,00 & 12 & 30 & 40 & 100 \\
\hline
\end{tabular}

Tabla 6. Polifarmacia y calidad de vida

\begin{tabular}{lccc}
\hline & \multicolumn{3}{c}{ Calidad de vida } \\
\cline { 2 - 4 } & Función física & $\begin{array}{c}\text { Bienestar } \\
\text { emocional }\end{array}$ & $\begin{array}{c}\text { Función } \\
\text { social }\end{array}$ \\
\hline Sin Polifarmacia & 66 & 68 & 81 \\
Polifarmacia Menor & 57 & 56 & 71 \\
Polifarmacia Mayor & 44 & 58 & 70 \\
Promedio & 56 & 61 & 74 \\
\hline
\end{tabular}


Tabla 7. Adherencia farmacológica y Calidad de vida

\begin{tabular}{lccc}
\hline & \multicolumn{3}{c}{ Calidad de vida } \\
\cline { 2 - 4 } & $\begin{array}{c}\text { Función } \\
\text { física }\end{array}$ & $\begin{array}{c}\text { Bienestar } \\
\text { emocional }\end{array}$ & $\begin{array}{c}\text { Función } \\
\text { social }\end{array}$ \\
\hline Adherencia Total & 46 & 58 & 72 \\
Adherencia Parcial & 53 & 61 & 67 \\
Promedio & 50 & 60 & 70 \\
\hline
\end{tabular}

\section{DISCUSIÓN}

Según los objetivos de la investigación, se determinó la frecuencia de polifarmacia habiendo encontrado un valor de $92,5 \%$ en adultos mayores en el Centro Asistencial de la Sociedad de Beneficencia de Huancayo, correspondiendo a polifarmacia mayor $72,50 \%$ y polifarmacia menor un $20 \%$, estos valores alto de polifarmacia coinciden con el estudio de Robles y col., en su investigación sobre calidad de vida y polifarmacia del adulto mayor integrante del programa adultos mayores empacadores, donde determinaron una frecuencia de polifarmacia de $75,2 \%$ siendo diferente en cuanto al nivel de polifarmacia ya que en su estudio el $68,8 \%$ con polifarmacia menor, y el 6,4\% con polifarmacia mayor (7). Asimismo, los estudios de Serra y Germán en la investigación Caracterización de adultos mayores con polifarmacia evaluados en la consulta de geriatría considerando como polifarmacia la administración simultánea de tres fármacos a más, los resultados fueron una elevada polifarmacia (8), por lo tanto, la polifarmacia en el adulto mayor se debe a las múltiples enfermedades padecen y deben tener un tratamiento para cada una de ellas.

En la tabla 2 los resultados muestran que el grupo farmacológico más prescrito fueron las Vitaminas y minerales con un $29 \%$, seguido de los medicamentos psicoterapéuticos con un $17 \%$, los medicamentos cardiovasculares con un $14 \%$, los medicamentos que actúan en las vías respiratorias con $7 \%$ y los analgésicos con un $6 \%$, resultados que difieren con la investigación de Serra y Germán quienes hallaron que los medicamentos cardiovasculares fueron los más prescritos con un $86,9 \%$, seguidas de los medicamentos osteoarticulares con un $75,8 \%$ y las endocrinas metabólicas con 47,5\% (8); así mismo difiere con los resultados de Zavala et al., quienes concluyen que el grupo farmacológico más prescrito fueron los analgésicos, seguido de los medicamentos cardiovasculares (9).
Con respecto a la adherencia farmacológica el estudio presenta $100 \%$ de adherencia, estos resultados se deben a que los adultos mayores son de un centro residencial el que cuenta con personal especializado para la administración de los medicamentos en el horario y dosis correspondiente a cada uno, de este $100 \%$ la adherencia total corresponde al $70 \%$ (34 a 48 puntos) y adherencia parcial $30 \%$ (18 a 33 puntos), lo que difieren con los resultados de García, que encontró un rango de adherencia media de $53 \%$ y en un alto nivel de adherencia 47\% (10).

Referente a la calidad de vida de los adultos mayores se ha encontrado un valor global de 58,23, lo que indica, que la calidad de vida se encuentra en un estado regular, según la escala de percentiles. Asimismo, de las dimensiones de funcionamiento social se encontró un valor 70,63 indicando un estado regular, el funcionamiento físico en estado malo con un valor de 47,85 y bienestar emocional en estado regular con un valor de 58.08, estos resultados coinciden con los de Mendoza et al., en su investigación, donde exploran sobre el Impacto de la polimedicación y su influencia en la calidad de vida de adultos mayores que se encuentran en un centro geriátrico donde la dimensión más afectada fue el funcionamiento físico $: 31,41$, los mejores promedios se obtuvieron en el funcionamiento social 79,16, y rol emocional 76,06 (11). Coincidente también con los estudios de Robles et al., en su investigación Calidad de vida y polifarmacia del adulto mayor integrante del programa adultos mayores empacadores, cuyos resultados en Salud física fueron de un $62,72 \%$, psicológico $62,78 \%$ y relaciones interpersonales $63,89 \%$ (7).

En la aplicación de la prueba estadística de independencia Chi cuadrado para la evaluación de la relación entre variables se encontró que no existe relación entre polifarmacia y adherencia farmacológica, si existiendo una relación entre polifarmacia y calidad de vida, encontrándose también 
una relación entre adherencia farmacológica y calidad de vida, no se han encontrado estudios que relacionen las variables mencionadas.

Debemos considerar que los cambios fisiológicos en el adulto mayor alteran el metabolismo de los medicamentos y la polimedicación aumenta el riesgo de sufrir reacciones adversas e interacciones medicamentosas en función al número de fármacos administrados afectando la calidad de vida.

\section{CONCLUSIONES}

La polifarmacia es un problema de salud pública, que tiene una frecuencia elevada del 92,5\% y tiene una relación inversa con la calidad de vida de los adultos mayores, debido que los cambios fisiológicos en el adulto mayor alteran el metabolismo de los medicamentos y la polimedicación aumenta el riesgo de sufrir reacciones adversas e interacciones medicamentosas en función al número de fármacos administrados debido. principalmente por padecer de múltiples enfermedades, por lo tanto, es necesario establecer políticas de salud destinadas a la atención adecuada de este grupo etario para mejorar su calidad de vida la cual se encuentra en un nivel regular.

La adherencia al tratamiento farmacológico es fundamental para disminuir las consecuencias de la polifarmacia como son las reacciones adversas e interacciones farmacológicas, en el centro residencial de estudio la adherencia es al $100 \%$ debido a que la administración de los medicamentos es controlada por profesionales.

\section{Correspondencia:}

Mónica Alejandra Calle Vilca

Correo electrónico: mcalle@uroosevelt.edu.pe

\section{REFERENCIAS BIBLIOGRÁFICAS}

1. Bjerrum L, Rosholm J, Hallas J, Kragstrup J. Methods for estimating the occurrence of polypharmacy by means of a prescription database. Eur J Clin Pharmacol. 1997;53(1):7-11. DOI: 10.1007/s002280050329

2. Organización Mundial de la Salud. Informe Mundial Def. de Polifarmacia. Ginebra: Organización Mundial de la Salud; 2013

3. Veehof L, Stewart R, Haaijer-Ruskamp F, Jong B. The development of polypharmacy. A longitudinal study. Fam Pract. 2000;17(3):261-7. DOI: 10.1093/ fampra/17.3.261.
4. The World Health Organization Quality of life assessment (WHOQOL). Position Paper from the World Health Organization. Social Science \& Medicine. 1995; 41 (10): 1403-09.

5. World Health Organization. Adherence to long term therapies: evidence for action. Ginebra: World Health Organization; 2003

6. Nieto M, Abad M, Torres A. Dimensiones psicosociales mediadores de la conducta de enfermedad y la calidad de vida en población geriátrica. Anales de psicología. 1998; 14(1):75-85.

7. Robles R, Hernández E, Delabra M, Covarrubias I, Leija A, Ponce B. Calidad de vida y polifarmacia del adulto mayor integrante del programa "adultos mayores empacadores". Nure Investigación. 2017; 14(91). (Citado el 3 de febrero del 2021). Disponible en: https://www.nureinvestigacion.es//OJS/index. $\mathrm{php} /$ nure/article/view/1206

8. Serra M, Germán J. Caracterización de adultos mayores con polifarmacia evaluados en la consulta de geriatría. Rev Cub Med Mil. 2014; 43(3):285-292. (Citado el 3 de febrero del 2021). Disponible en: http:// scielo.sld.cu/scielo.php? script=sci_arttext\&pid= S0138-65572014000300003\&lng=es

9. Zavala J, Terán M, Nava M, Pinedo M, De La Mata M. Detección de polifarmacia y prescripción potencialmente inapropiada en el adulto mayor en una unidaddemedicinafamiliar.AtenFam.2018;25(4):141145. DOI: 10.22201/facmed.14058871p.2018.4.6725 7

10. García M. Factores relacionados con la Adherencia al tratamiento farmacológico y no farmacológico en pacientes con post infarto agudo de miocardio en una institución de cuarto nivel, Bogotá durante el mes de agosto de 2008. Tesis de grado. Bogotá: Pontificia Universidad Javeriana; 2008. (Citado el 3 de febrero del 2021): Disponible en: https://repository.javeriana. edu.co/bitstream/handle/10554/9444/Tesis01.pdf;seq

11. Mendoza L, Reyna N, Bermúdez V, Nuñez J, Linares $\mathrm{S}$, Nucette L, et al. Impacto de la polimedicacion en la calidad de vida de adultos mayores institucionalizados en un centro geriatrico del estado Zulia. Archivos Venezolanos de Farmacología y Terapéutica. 2016; 35(2):47-52. (Citado el 3 de febrero del 2021): Disponible en: http://www. redalyc.org/articulo.oa? $\mathrm{id}=55949906003$

Recibido: 24/03/2021

Aceptado: 18/07/2021 